\title{
A case of retinal damage caused by green laser pointer
}

\begin{abstract}
We report the case of a 16-year-old boy who developed central scotoma a month after staring at the light beam of a laser pointer device. Duration time was 3-6 seconds and the device was classified as IIIA green laser pointer with less than $5 \mathrm{~mW}$ power. Clinical examination revealed normal corrected visual acuity and paracentral scotoma in the left eye with focal disruption of inner/outer segment junction of photoreceptor and mild thinning of Retinal Pigmented Epithelium (RPE) of left macula in Optical Coherence Tomography $(\mathrm{OCT})$. He was treated with oral corticosteroids and significant improvement was observed after 2 months of follow up.
\end{abstract}

Keywords: retinal damage, visible light, laser pointer
Volume 2 Issue 5 - 2015

\author{
Ramin Farajpour, ${ }^{1,2}$ Behzad Fallahi, ${ }^{3}$ Shiva \\ Farajpour, ${ }^{4}$ Masoud Bagheri, ${ }^{4}$ \\ 'Neuroscience Research Center (NSRC), Tabriz University of \\ Medical Sciences, Iran \\ ${ }^{2}$ Student Research Committee, Tabriz University of Medical \\ Sciences, Iran \\ ${ }^{3}$ Assistant professor, department of ophthalmology, Nikookari \\ Eye Hospital, Tabriz University of Medical Sciences, Iran \\ ${ }^{4}$ Resident of ophthalmology, Ophthalmic Research Center, \\ Nikookari Eye Hospital,Tabriz University of Medical Sciences, \\ Iran
}

Correspondence: Shiva Farajpour, Resident of ophthalmology, Nikookari Eye Hospital, Tabriz University of Medical Sciences, Tabriz, East Azarbayjan, Iran, Tel +989397368408, Fax +984|36577336, Email dr.farajpur@gmail.com

Received: April 13, 2015 | Published: July 31, 2015
Abbreviations: FDA, food and drug administration; RPE, retinal pigmented epithelium; OCT, optical coherence tomography

\section{Introduction}

In recent years, laser pointers are used in various fields, especially for educational purposes. They are also used frequently by children as toys. These devices are consisted of laser diodes, emitting a very narrow coherent laser beam of visible light. Food and Drug Administration (FDA) has categorized lasers into four classes and laser- pointers are placed in class IIIA. According to this category, direct exposure to class III lasers can result destructive ophthalmic effects. Green laser pointers are commonly known as safe devices because of lower degree of power; however there remains a debate among ophthalmologists about their potential to cause retinal damage. In this report, we present a case of retinal damage induced by a green laser pointer in a 16-year- old teenager.

\section{Case presentation}

A 16-year-old boy was referred to our out-patient clinic. He was complaining of a central black spot in his left eye. The patient was directly exposed to the beam of a class IIIA green laser pointing device about a month ago. He has starred for 3-6 seconds to light beam. The device was used as toy pointer with the maximum power rating of 50 $\mathrm{mv}$ and wavelength of $532 \pm 10 \mathrm{~nm}$. He flashed the laser light from the distance of $25 \mathrm{~mm}$ away from Cornea and gazed only one time to the light. The patient's history revealed no visual complaints, prior to this event. Clinical examination revealed visual acuity correctness of 10/10 in the right eye and 10/10 in the left eye. Anterior segments of the both eyes were intact in the examination. Fundus examination showed a yellowish dot lesion in fovea of the left retina (Figure 1).

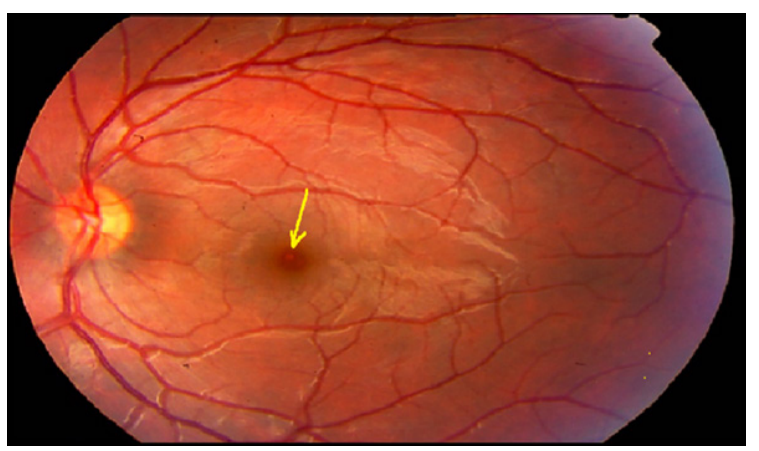

Figure I Fundus photographs showing yellowish point in the left fovea.

In addition, 30-2 Threshold Visual Field Tests with Standard Automated Perimetry, found paracentral scotoma in the left eye. In the red-free fundus photographs, heterogeneous foveal lesions were detected in the left retina (Figure 2). Optical coherence tomography (OCT) of the affected eye showed focal disruption of inner/outer segment junction of photoreceptor and mild thining of Retinal Pigmented Epithelium (RPE) of left macula. Retinal photoreceptor layer was disrupted, also mild reduction in the pigmented epithelium diameter was detected (Figure 3). The patient was treated with corticosteroid. Tapering doses of oral prednisolone $(40 \mathrm{mg} /$ day) was administered for 3 weeks. He was on 2 months follow up after the first ophthalmic visit and visual examination revealed great improvement in the later months. 


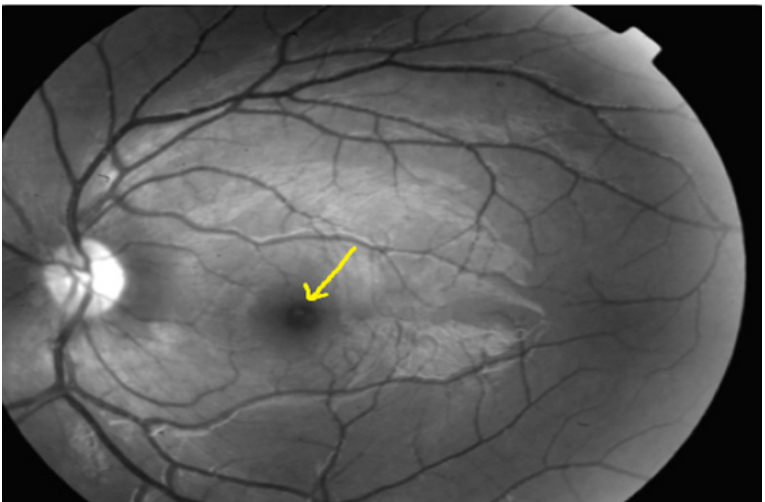

Figure 2 Red-free fundus photographs showing heterogeneous foveal lesions.

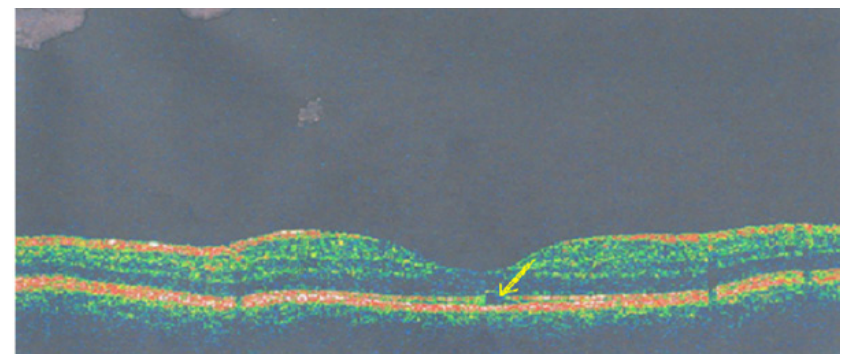

Figure 3 Optical Coherence Tomography (OCT): focal disruption of inner/ outer segment junction of photoreceptor and mild thickening of Retinal Pigmented Epithelium (RPE) of left macula.

\section{Discussion}

Nowadays laser pointers are readily available. Previously, laser pointers were considered as safe and harmless devices, but recent literature reports presented a few cases of pointer-induced retinal damage. These devices have become popular among young people for their novel application in lighting cigarettes, popping balloons, and burning holes in objects also among children as a toy. This may lead to a public concern about the potential harms of these devices. In addition, an increasing number of reports of retinal damage caused by laser pointer devices have increased this concern. Varying pathologies caused by handheld laser pointers are described in these reports.

According to Barkana \& Belkin, ${ }^{1}$ several factors contribute to laser-related retinal damage, and these can be divided into two categories, i.e, laser-related factors (wavelength of the radiation; pulse duration; and energy of the beam) and patient-related factors (size of the pupil, with injury being more severe in larger pupil sizes; degree of retinal pigmentation, with dark-skinned individuals suffering more severe injury than light-skinned ones; proximity of incident beam to the fovea; and refraction status, with damage being more severe in emmetropic eyes due to the laser beam being more focused on the retina). Also, experimental studies that have evaluated the clinical and histopathologic effects of laser pointers on eyes undergoing enucleation for melanoma concluded that green laser pointers (490-575 nm) are more damaging to the retina than red laser pointers $(635-750 \mathrm{~nm}){ }^{2,3}$ Probable other factor in laser-related retinal damage is the age of the patient. Laser-induced damage to the retina is even more concerning in children and infants than adults. Where as adults terminate accidental laser pointer exposure in less than 0.25 seconds by pupil, blink, and aversion responses, ${ }^{4}$ children have been reported to display "unusual" behavior i.e, staring for a prolonged period of time at the laser beam without blinking or averting the eye. ${ }^{5}$. The severity of damage is directly dependent on the laser type, wavelength, duration of exposure, and spot size. Different ocular structures absorb light of varying wave lengths, with photochemical retinal damage (photocoagulation and photo disruption) more likely to be caused by blue light. ${ }^{6}$

Laser pointers are coded by wavelength, power and continuous or pulsatile light flashing. The classification according to the laser power is listed below:

1. Class I (low output power): no possibility of retinal damage even after hours of exposure.

2. Class II (output power less than $1 \mathrm{mw}$ ): lasers with visible light including most of the laser pointers are included in this group.

3. Class II a (output power less than $1 \mathrm{mw}$ ): The pointers in this category might be harmful by excess of 1,000 seconds of continuous exposure.

4. Class III A (output power between 1 and $5 \mathrm{mw}$ ): The device is not labeled with a "caution" warning, and the possible damages are controversial. Many laser pointers are put in this category.

5. Class III b (Output power between 5-500 mw): Lasers in this category are capable to cause permanent retinal injury with exposures of $1 / 100$ of a second.

6. Class IV (output power more than 500): all the lasers in this category can cause severe, permanent damage to eye even by a short glance.

The most popularly used laser pointers are categorized in class III A or class II. In contrast, class III b devices are commonly used by ophthalmologists to perform retinal operations. ${ }^{7,8}$ Laser pointers with an output power of less than $5 \mathrm{~mW}$ are regarded as harmless to the human eye because of the limited exposure aided by innate ocular protective mechanisms (i.e blink reflex and aversion response). ${ }^{9}$ Devices with an output power of more than $150 \mathrm{~mW}$ are capable of causing severe ocular damage. ${ }^{10,11}$ Retinal damage from high-power lasers has been reported mostly in military, industrial, or medical use settings. ${ }^{7}$ Most of the reported data support the retinal hazards of class III A green laser pointers. These damages varied from small retinal lesions to chorioretinal hemorrhage or rarely choroidal neovascularization.

In this report, the left eye of a young patient was hurt by a handheld green laser pointer. Despite the absence of recognized changes in visual acuity, there were abnormalities of the outer layer of retina of the affected eye. In the first reported cases of photo toxicity from handheld laser pointers, the lasers were Class 3 a lasers emitting a red light of $632 \mathrm{~nm} \cdot{ }^{2,13-16}$ More recently, cases of retinal injury from green laser have been reported. ${ }^{17,18}$

In 1999, report of a pointer-induced retinal damage was published, in which the patient was presented with central scotoma and transient headache, while visual acuity didn't change after the exposure. ${ }^{15}$ On the other hand, another case reported in the same year revealed a reduced visual acuity in both eyes with loss of the central foveal reflex. ${ }^{16}$ Additional cases of probable retinal damage were studied by Robertson et al. ${ }^{2}$ in 2000 . The cases were presented as a 62 -yearold man, a 36-year-old woman and a 59-year-old woman with uveal melanomas, who were scheduled to have an enucleation. The patients 
underwent the continuous laser beams from commercially available class IIIA laser pointers and any recognizable retinal damages were evaluated. It was concluded the potential risk of the class IIIA laser pointers to cause eye damages is negligible. These findings were also confirmed in the survey of 14 patients with transient exposure to light beam of a laser pointer. ${ }^{19}$ Regardless of the previous reports, the vision-threatening hazards of these devices, especially class IIIA pointers have been proven in different reports..$^{20-23}$

Wyrsch et al. ${ }^{10}$ reported a 15 -year-old boy who sustained a sub macular hemorrhage after using a handheld green laser pointer with an output power of $150 \mathrm{~mW}$. Similar findings were recently published by Alsulaiman et al ${ }^{11}$ after the use of handheld lasers with $750 \mathrm{~mW}$ output power and $450 \mathrm{~nm}$ wavelengths. In 2013 the first case report that demonstrated laser-induced retinal injury documented by both spectral domain optical coherence tomography and fundus autofluorescence was presented by Irene Rusu et al. ${ }^{24}$ in that report, optical coherence tomography images showed that the ellipsoid zone and external limiting membrane are disrupted at the site of the lesion. In addition, they report reversible outer retinal disruption overlying the RPE similar in appearance to outer retinal tabulation.

Outer retinal disruption, epiretinal membrane with subretinal fluid, and unilateral macular holes were also presented in this series were associated with a shorter ocular-laser exposure distance. Petros Petrou et al. ${ }^{25}$ reported bilateral macular holes caused by the use of a handheld laser pointer in a patient with bilateral injury occurred when he was attempting to light a cigarette with simultaneous foveal exposure. Dishler S Dhoot et al. ${ }^{26}$ reported high-powered laser pointer injury resulting in macular hole formation in a 16-year-old boy patient. The laser pointer used in this case emitted $1000 \mathrm{~mW}$, an energy level approximately 200 times greater than the threshold for normal Class $3 \mathrm{~A}$ pointers. These hazards were presented as permanent reduction in visual acuity due to macular damage in most of the cases and more common in children and teenagers than the adults. Changes in the retinal pigmented epithelium were also common among the reported cases.

Current treatment for the macular injuries induced by laser beam is mainly limited to corticosteroids, but final visual recovery may be partial regarding the lesion size and location. ${ }^{1}$ Final visual acuity ranges from 20/20 to 20/60 vision, and this depends on the size and location of the macular lesion. ${ }^{27}$ Our patient was also treated with oral prednisolone and the significant visual improvement was observed in the next ophthalmologic visits. This report indicates the danger of laser pointers to cause serious visual problems. So the ophthalmologists and the public should be aware of the potential hazard of these devices. The potential vision-threatening hazards caused by mishandling laser pointers, even class $3 \mathrm{~A}$ lasers, emphasize the importance of cautious and appropriate use of these devices. Recommendations regarding the purchase and use of these devices most are reconsidered. Further restrictions on their sale and use by the general public will require more than simple recommendations; legislation will have to be passed and enforced by governmental bodies.

\section{Funding}

None.

\section{Acknowledgments}

We are thankful to Dr Rana Sorkhabi for spiritual auspices and recommendation of this journal for submitting our article.

\section{Conflicts of interest}

The authors have no conflicts of interest to declare.

\section{References}

1. Barkana Y, Belkin M. Laser eye injuries. Surv Ophthalmol. 2000;44(6):459-478.

2. Robertson DM, Lim TH, Salomao DR, et al. Laser pointers and the human eye: a clinicopathologic study. Arch Ophthalmol. 2000;118(12): 16861691.

3. Robertson DM, McLaren JW, Salomao DR, et al. Retinopathy from a green laser pointer: a clinicopathologic study. Arch Ophthalmol. 2005;123(5):629-633

4. Mainster MA, Timberlake GT, Warren KA, et al. Pointers on laser pointers. Ophthalmology. 1997;104(8):1213-1214.

5. Fujinami K, Yokoi T, Hiraoka M, et al. Choroidal neovascularization in a child following laser pointer-induced macular injury. Jpn J Ophthalmol. 2010;54(6):631-633.

6. Ham WT, Ruffolo JJ, Mueller HA, et al. Histologic analysis of photochemical lesions produced in rhesus retina by short-wave-length light. Invest Ophthalmol Vis Sci. 1978;17(10):1029-1035.

7. Bren L. Illuminating facts about laser pointers. FDA Consum. 2005;39(3):18-19.

8. Nakagawara VB, Montgomery RW. Laser pointers and aviation safety. Aviat Space Environ Med. 2000;71(10):1060-1062.

9. Mainster MA, Stuck BE, Brown J. Assessment of alleged retinal laser injuries. Arch Ophthalmol. 2004;122(8):1210-1217.

10. Wyrsch S, Baenninger PB, Schmid MK. Retinal injuries from a handheld laser pointer. $N$ Engl J Med. 2010;363(11):1089-1091.

11. Alsulaiman SM, Alrushood AA, Almasaud, et al. High-power handheld blue laser-induced maculopathy: the results of the King Khaled Eye Specialist Hospital Collaborative Retina Study Group. Ophthalmology. 2014;121(2):566-572.

12. Ciulla TA, Topping TM. Surgical treatment of a macular hole secondary to accidental laser burn. Arch Ophthalmol. 1997;115(7):929-930.

13. Zamir E, Kaiserman I, Chowers I. Laser pointer maculopathy. Am $J$ Ophthalmol. 1999;127(6):728-729.

14. Isralei D, Hod Y, Geyer O. Laser pointers: not be taken lightly. $\mathrm{Br} J$ Ophthalmol. 2000;84(5):555-556.

15. Luttrull JK, Hallisey J. Laser pointer-induced macular injury. Am J Ophthalmol. 1999;127(1):95-96.

16. Sell CH, Bryan JS. Maculopathy from handheld diode laser pointer. Arch Ophthalmol. 1999;117(11):1557-1558.

17. Ziahosseini K, Doris JP, Turner GS. Laser eye injuries. Maculopathy from handheld green diode laser pointer. BMJ. 2010;340:c2982.

18. Ueda T, Kurihara I, Koide R. A case of retinal light damage by green laser pointer (class 3b). Jpn J Ophthalmol. 2011;55(4):428-430.

19. Sethi CS, Grey RH, Hart CD, Laser pointers revisited: a survey of 14 patients attending casualty at the Bristol Eye Hospital. B J Ophthalmol. 1999;83(10):1164-1167.

20. Lally DR, Duker JS. Foveal injury from a red laser pointer. JAMA Ophthalmol. 2014;132(3):297.

21. Keunen JE, Delbecq AM, Cruysberg JR, et al. Laser pointers are not toys; eye injury with permanent loss of visual acuity. Ned Tijdschr Geneeskd. 2013;158:A7813. 
22. Dirani A, Chelala E, Fadlallah A, et al. Bilateral macular injury from a green laser pointer. Clin Ophthalmol. 2013;7:2127-2130.

23. Pollithy S, Ach T, Schaal KB, et al. Acute bilateral impaired vision with central scotoma in an 11-year-old boy. Ophthalmologe. 2012;109(9):907910.

24. Rusu I, Sherman J, Gallego-Pinazo R, et al. Spectoral-domain Optical Coherence Tomography and Fundus Autofluoresence Findings in a case of Laser Pointer-induced Maculopathy. Retin Cases Brief Rep. 2013;7(4):371-376
25. Petrou P, Patwary S, Banerjee PJ, et al. Bilateral macular hole from a handheld laser pointer. Lancet. 2014;383(9930):1780.

26. Dhoot DS, $\mathrm{Xu} \mathrm{D}$, Srivastava S. High-Powered Laser Pointer Injury Resulting in Macular Hole formation. J Pediatr. 2014;164(3):668.

27. Turaka K, Bryan JS, Gordon AJ, et al. Laser pointer induced macular damage: case report and mini review. Int Ophthalmol. 2012;32(3):293297. 